performed in two steps; first on CAs previously associated with seasonal factors and second on all remaining CAs. CAs and monthly births were calculated back to month of last menstrual period after which trigonometric regression analysis was performed to explore seasonal trends in CA prevalence.

Our dataset confirmed seasonality for Ebstein's anomaly $(p<0.05)$, triscuspid atresia and stenosis $(p<0.05)$, congenital hydronephrosis $(p<0.001)$ and hip dislocation $(p<0.001)$ and a new signal was generated for seasonality of situs inversus $(p<0.001)$. We detected non-significant seasonal peaks for neural tube defects $(\mathrm{p}=0.0683)$ and spina bifida $(\mathrm{p}=0.0507)$ coinciding with influenza season. We were not able to detect seasonality for any other CAs. We were unable to confirm the associations between neural tube defects, some other anomalies and influenza.

The associations detected and the negative results provided can help future studies unravelling the etiology of CAs.

\section{MOLECULAR ETIOLOGY OF CHILDHOOD HEARING IMPAIRMENT ASSOCIATED WITH NON-SYNDROMIC ENLARGED VESTIBULAR AQUEDUCT IN SOUTHEASTERN CHINA}

doi:10.1136/archdischild-2012-302724.0251

1,2T Yang, 1,2Y Chai, 1,2 H Wu. 'Department of Otolaryngology - Head and Neck Surgery, Xinhua Hospital, Shanghai Jiao Tong University School of Medicine; '2Ear Institute, Shanghai Jiao Tong University School of Medicine, Shanghai, China

Background Mutations in SLC26A4, and in rarer cases double heterozygous mutations of FOXI1/SLC26A4 or KCNJ10/SLC26A4, lead to childhood hearing impairment associated with non-syndromic enlarged vestibular aqueduct (EVA), the most common inner ear malformation. Molecular etiology studies of non-syndromic EVA will provide important data to facilitate DNA diagnosis and genetic counseling of this disease.

Methods Mutation screening of SLC26A4 was performed in 126 probands with non-syndromic EVA in Southeastern China. Those detected with mono-allelic or no SLC26A4 mutation were subjected to mutation screening of FOXI1 and KCNJ10.

Results Bi-allelic, mono-allelic, and no SLC26A4 mutation were detected in $70.6 \%, 8.0 \%$ and $21.4 \%$ of the probands with non-syndromic EVA. Sixteen of the 40 SLC26A4 mutations detected were novel. While the c.919-2A>G mutation accounted for $41.3 \%$ of the mutant alleles of $S L C 26 \mathrm{~A} 4$, none of the other 39 mutations accounting for more than $5.6 \%$. No pathogenic FOXI1 or KCNJ10 mutation was identified in this study.

Conclusions The c.919-2A>G mutation of SLC26A4 is highly prevalent and should be the primary target of genetic testing for patients with non-syndromic EVA in Southeastern China. The spectrum of the other SLC26A4 mutations, however, is highly heterogeneous and differs from those reported in Taiwan or other regions of mainland China. Mutations in FOXI or KCNJ10 were not the major cause of non-syndromic EVA in Southeastern China.

\section{WHOLE GENOME MICRORNA EXPRESSION PROFILING IN CHILDHOOD ACUTE LYMPHOBLASTIC LEUKEMIA: A PROSPECTIVE EVALUATION}

doi:10.1136/archdischild-2012-302724.0252

${ }^{1} \mathrm{M}$ Duyu, ${ }^{1} \mathrm{O}$ Cogulu, ${ }^{1 B}$ Durmaz, ${ }^{1} \mathrm{C}$ Gunduz, ${ }^{2} \mathrm{C}$ Vergin, ${ }^{1} \mathrm{D}$ Yilmaz Karapinar, ${ }^{1} \mathrm{~S}$ Aksoylar, ${ }^{1} \mathrm{~K}$ Kavakli, ${ }^{1} \mathrm{~N}$ Cetingul, ${ }^{3} \mathrm{G}$ Irken, ${ }^{2} \mathrm{Y}$ Yaman, ${ }^{1} \mathrm{~F}$ Ozkinay. ${ }^{1}$ Ege University Faculty of Medicine; ' ${ }^{2}$ Behcet Uz Children Hospital; ${ }^{3}$ Faculty of Medicine, Dokuz Eylül University, Izmir, Turkey

The aim of the study is to evaluate the associations between micro RNAs (miRNAs) and childhood acute lymphoblastic leukemia (ALL). Forty-three children with ALL and 14 age-matched controls were included in the study. Microarray expression profiling consisting of 1136 miRNAs was performed in peripheral blood and bone marrow samples of patients. Diagnosis, differential diagnosis, outcome and prognosis associated with aberrant microRNA expressions were prospectively evaluated. Significant miRNAs on admission were confirmed and re-evaluated after 6 months following treatment period by real time RT-PCR. The effect of miRNAs on overall survival (OS) and event free survival were presented. The most significantly upregulated miRNAs were miR-548i (12.5 fold), miR-708 (10 fold), miR-181b (6.25 fold) and most downregulated miRNAs were miR-145 ( -2.52 fold) and miR-640 ( -2.3 fold) compared to control group in microarray profiling. miRNAs according to immunophenotype revealed 22 upregulated and 13 downregulated in T-ALL. In the B-lineage ALL group, 7 miRNAs were upregulated and 2 miRNAs were downregulated. Expression of miR-146a, miR-155, miR-181a and miR-195 significantly changed after 6 months of treatment period. miR-145 was associated with OS. $\mathrm{t}(12$ $21)$ and $t(9 ; 22)$ were significantly associated with certain miRNAs. In conclusion miRNA expression profile could be used as biomarker in the diagnosis, differential diagnosis, monitoring the disease and prognosis of ALL.

\section{MANAGEMENT OF HEMANGIOMAS: A SINGLE CENTER EXPERIENCE}

doi:10.1136/archdischild-2012-302724.0253

G Tuysuz, N Ozdemir, B Kutlubay, T Celkan. Pediatric Hematology-Oncology Dept., Istanbul University, Cerrahpasa Medical Faculty, Istanbul, Turkey

Aim To share our experience of 185 (F/M: 2.4) patients with hemangiomas followed between 2003-2011 at Cerrahpasa Medical Faculty, Istanbul.

Results Onehundred-twenteight (69\%) had a single lesion whereas $31 \%$ had multiple lesions. Most of the patients (n:94) were followed by a "watch and wait" policy and 40 of them did not require any medical or surgical intervention as the lesion convoluted on followup. Only $6(3 \%)$ patients required surgery. Nine patients with lesion around eye required intralesional steroids to prevent visual disturbance. Bleomycin was used as a sclerosing treatment in 2 patients with a giant hemangioma. In 21 patients with multiple diffuse lesions causing cosmetic problems, interferon was given. Medical treatment was given in other patients on follow-up due to growth, ulceration, bleeding or persistance of the lesions at older ages; 14 were treated by systemic steroids, 73 by propranolol and 39 by combination therapy due to insuffient response. Propranolol was the first choice of treatment in patients diagnosed after 2008. All patients treated by propranolol were evaluated by echocardiography and electrocardiograpy, no cardiac side effects were noted. One patient under treatment presented with increased sweating and was found to have hypoglycemia associated with propranolol during periods of restricted oral intake. The drug was restarted increasing the frequency of breastfeeding with no further hypoglycemia attacks.

Conclusion The excellent clinical outcome and apparent lack of side effects of propranolol makes it a good choice as a first-line treatment for hemangiomas. Hypoglycemia may be noted in infants under propranolol in restricted periods of feeding.

\section{NEONATAL BLOOD TRANSFUSION-CAN WE MAKE IT SAFER?}

doi:10.1136/archdischild-2012-302724.0254

LA Funston, M Hogan. Paediatric Department, Southern Health and Social Care Trust, Portadown, UK

Background and Aims A specific blood transfusion booklet was implemented in the Southern Health and Social Care Trust in 2010 for babies less than four months of age. This aimed to provide a 